\title{
Some mathematical aspects on walking robots stable evolution
}

\author{
Marcel Migdalovici, Luige Vladareanu*, \\ Gabriela Vladeanu \\ Robotics and Mechatronics Department \\ Romanian Academy, Institute of Solid Mechanics \\ Bucharest, Romania, luigiv@arexim.ro
}

\section{Daniela Baran}

Dynamical Systems, National Institute of Aircraft Research "Elie Carafoli", Bucharest, Romania

dbaran@incas.ro

\author{
Said Broumi \\ Laboratory of Information processing, \\ Faculty of Science Ben M'Sik, University Hassan II, B.P 7955, \\ Sidi Othman, Casablanca, Morocco, broumisaid78@gmail.com \\ Florentin Smarandache \\ Department of Mathematics, University of New Mexico, \\ 705 Gurley Avenue, \\ Gallup, NM 87301, USA \\ fsmarandache@gmail.com
}

\author{
Prof. Hongbo Wang, Yonfei Feng, \\ Parallel Robot and Mechatronic System Laboratory of Hebei Province \\ Key Laboratory of Advanced Forging \& Stamping Technology and Science of Ministry of Education \\ Yanshan University, Qinhuangdao, China \\ hongboww@ysu.edu.cn
}

\begin{abstract}
A survey of some author's concepts on the dynamic systems stability regions, in the general case of dynamic systems that depend on parameters is related in the paper. The property of separation of stable regions in the free parameters domain is assumed in the paper as an important property of the environment that is carry out and in the specified case of walking robot analyzed in the paper. The matrix that defines the linear dynamic system has the components of the matrix, assumed to be with real values, and the matrices that intervene in the exposure of the method are also, with real values of the components. We assumed that the matrices from the exposure have the complex values such that the real values are also taken into account as particular case of the complex values. This hypothesis assures a new method of analysis, in the complex domain, on the dynamic systems stability. Our theory on the stability control of the dynamic systems is applied here for specified walking robot model that depend on parameters. The critical position of the walking robot evolution is defined and analyzed on some cases of the walking robot leg, and possible application for robot walking up stairs is exposed. The further way of research is emphasized.
\end{abstract}

Keywords: dynamic system; stable region; walking robots; critical position; walking up stair; kinematics analyze

\section{INTRODUCTION}

Any dynamic system can be considered as dynamic system in terms of its defining parameters without fixing their values as physical parameters (in particular mechanical parameters), geometrical parameters, possible economical, biological parameters or other.

An important idea is that many concrete dynamic systems from the literature that depend on parameters have the proper- -ty of separation between the stable and unstable regions in a specified domain of free parameters. The stable and unstable regions are separated in the domain of free parameters by a boundary. The property of separation can be defined by the fact that the stable and unstable regions are open sets, excepting the points on boundary. This separation aspect creates the freedom of stability control on a neighborhood of any stable point in the open stable region of the dynamic system. We discovered mathematical conditions of the stability regions existence for the dynamic systems using various results from applied mathematics domain.

The property of separation of stability regions is an important property of the environment that can contribute to mathematical modelling of it.

In this paper we analyze some aspects on the conditions of separation between stable and unstable regions in the free parameters domain of the linear or nonlinear dynamical systems, calling the QR algorithm applied on the real matrix that defines the linear dynamic system or on the "first approximation" of the nonlinear dynamic system, using operations in the complex domain by the matrix "shift of origin". The dependence of this matrix eigenvalues on the matrix components properties intervene in justification of separation property. The components of the real matrix $A$ that define the linear dynamic system depending on parameters are assumed to be piecewise continuous in the free parameters.

\section{On TRANSPOSITION OF THE REAL System IN COMPLEX ONE}

The linear dynamic system or the "first approximation" of the nonlinear dynamic system depending on some parameters

*Corresponding author: Luige Vladareanu 
is defined by one real matrix for the cases from the literature. The components of such real matrix are assumed to be piecewise continuous functions of the system parameters.

The properties of the stability for the dynamic system with parameters are described by the properties of eigenvalues functions for the system matrix. The eigenvalues of the system matrix are the same as for the matrix in Hessenberg corresponding form. The Hessenberg form is defined by the condition $a_{i j}=0$ for $2<i \leq n, j<i-1$. The dynamic system can be substituted by the dynamic system with the system matrix in Hessenberg form for the stability analysis of the system [28], [29].

The system matrix in Hessenberg form is denoted by $A$. The matrix $A-\lambda I$, where $\lambda$ is real or complex value, is also a matrix in Hessenberg form. The value $\lambda$ is named "shift of origin" of the matrix. The shift of origin for the matrix is important because allow the transposition of the real matrix that define the dynamic system in the complex one for analysis of the dynamic system stability.

The QR algorithm for matrix $A$, using "shift of origin", is defined by the relations [29]:

$$
Q_{s}\left(A_{s}-k_{s} I\right)=R_{s}, A_{s+1}=R_{s} Q_{s}^{T}+k_{s} I=Q_{s} A_{s} Q_{s}^{T}, s \in N
$$

In the above relations by $A_{1}$ is denoted initial matrix of the system in the Hessenberg form, $k_{s}$ is "shift of origin", $Q_{s}$ is orthogonal matrix, $R_{s}$ is upper triangular matrix, $A_{s}, s \geq 2$ is also in Hessenberg form and $N$ is natural numbers set.

The shift of origin, with the initial value $\lambda$ sufficient close to one initial matrix eigenvalue, real or complex, impose acceleration of the QR algorithm convergence to respective eigenvalue on the similar diagonal form of the matrix. This is an important property of QR algorithm with shift of origin.

In conclusion, the real matrix $A$ with distinct eigenvalues is similar with the matrix in Hessenberg form and QR algorithm with "shift of origin" can facilitate the convergence of the initial matrix to similar diagonal form of the matrix with real or complex eigenvalues on the diagonal.

This study is performed in the hypothesis that all eigenvalues of the real matrix are distinct. For extension of the results in the case of real matrix multiple eigenvalues, we called to the study of Hirsch, Smale and Devaney that have demonstrated that the set of matrices with distinct eigenvalues from linear normed space $L\left(R^{n}\right)$ is opened and dense set in this space [1].

The above remark gives the possibilities to motivate the transmission of some properties from the real matrices with distinct eigenvalues to the real matrices with multiple eigenvalues that can intervene in evolution analysis of the linear dynamic system.

\section{ON SEPARATION OF THE DyNAMIC System StABlE REGIONS}

Firstly we analyze the transmissibility of the piecewise continuity of the dynamic system matrix components depending of the system parameters to the eigenvalues of the matrix. This analysis is simplified in the case of the system real matrix substituted by the close matrix in the complex domain using "shift of origin".

Theorem 1. If the components of the matrix $A$ are piecewise continuous of the system parameters and the sequence of Hessenberg form matrices $A_{s}, s=1,2, \ldots$ from QR algorithm that started with the matrix $A$ is uniform convergent in the complex domain to the diagonal form of the matrix $A$ then the eigenvalues of the matrix $A$ are piecewise continuous.

This theorem is verified using the property that the uniform convergence of the continuous functions imposes the continuity on the function limit. In the case in which the eigenvalue is on the boundary between stable and unstable regions in the free parameters domain this eigenvalue has null real part and the character of the point (stable or unstable) is unknown. Above theorem is capitalized here using the following property formulated for the continuous functions of one variable.

Theorem 2. Let the function $f: E \rightarrow C, E \subset R$ where $R$ and $C$ are real respective complex domain, be a continuous function in the point $x_{0} \in E$ and the function value $f\left(x_{0}\right)$ with its module $\left|f\left(x_{0}\right)\right|$ so that the inequalities $\alpha<\left|f\left(x_{0}\right)\right|<\beta ; \alpha, \beta \in R$ are satisfied. Then there is a neighborhood of the point $x_{0} \in E$ where the function values carry out the same inequalities.

Remark: Theorem 2 assures that the function $f$, continuous in the point $x_{0} \in E$ preserve, in the neighborhood of $x_{0}$, the function module sign from $x_{0}$.

The mathematical conditions that assure the separation between stable and unstable regions for the linear dynamic system are described using and the following property:

Theorem 3. Let the linear dynamic system be defined by the differential equation of the form $d y / d t=A y(t)$,

$$
y(t)=\left(y_{1}(t), \ldots, y_{n}(t)\right)^{T}, \quad A=\left(a_{i j}\right), i=1, \ldots, n ; j=1, \ldots, n,
$$

where the symbol $T$ signifies the transposition of the matrix and the values $a_{i j}$ are assumed constants. If the real part of all eigenvalues of the matrix $A$ is strict negative then the solution of the differential equation is asymptotic stable in origin. If the real part of at least one eigenvalue of the matrix $A$ is strict positive then the solution of the differential equation is unstable in origin.

If the real parts of the matrix $A$ eigenvalues are strict negative with exception of at least one eigenvalue that has null real part then the stability of the dynamic system in origin is unknown (possible stable or unstable).

In the case of distinct eigenvalues of the real matrix $A$, attached to the linear dynamic system, a theorem on separation between stable and unstable regions in the free parameters domain of linear dynamic system, using the QR algorithm 
with the shift of origin in complex domain is formulated below. This theorem is deduced calling the theorems $1-3$.

Theorem 4 (Separation theorem). If the linear dynamic system defined by the real matrix $A$, in the Hessenberg form, has the piecewise continuous components of the matrix as functions of the dynamic system free parameters and the $Q R$ algorithm with the shift of origin in complex domain is convergent to the similar diagonal form corresponding to the matrix $A$ and assures that the real part of the eigenvalue functions from the similar diagonal form are also piecewise continuous, then these conditions determine the separation between stable and unstable regions of the dynamic system in the free parameters domain.

Remark: We comment on the possibility to substitute in practice the infinite QR algorithm by finite one and such that the conditions of the theorem 4 applications will be simplified.

In the following we analyze some problems of walking robot evolution by synchronization of their leg evolution in kinetics hypothesis. Extension to dynamic evolution of walking robot, on particular case, appealing to above theory is intuitive possible and is very attractive to study.

\section{Multi-LegGed WaLking Robot Modelling}

The physical and mathematical walking robot model, with physical model consists from a platform and four similar legs that have a joined extremity attached to the robot platform and a synchronized evolution in the vertical plane (fig.1).

Each of the leg is assumed to be compounded from two segments articulated between them and with this articulation, named and knee joint, traversing a circle arc route in a cycled evolution of the leg and where the base extremity of the leg traverse an imposed continuous route on the ellipse arc (fig.2). The leg base point in their evolution is assumed with constant speed component on horizontal direction, hypothesis that defines the leg evolution of the robot in our case studied. This physical model and corresponding mathematical model of the robot leg create the possibility to remark the possible appearance of the critical position, defined by the authors, on the circle arc of the knee joint assumed to have a trajectory on the circle arc in cyclic evolution of the walking robot leg. The points that specify the extremities of the continuous domain on

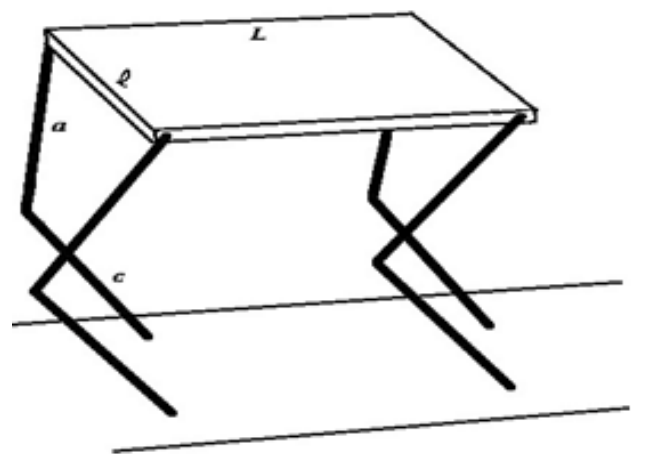

Fig.1 Walking robot physical model. circle arc where the knee joint is moving are defined in positions where the inferior segment of the leg is normally on the ellipse arc. The possibility to apply the identified critical positions of the walking robot evolution to climbing by stairs is mentioned in the paper.

Physical model of the walking robot leg is described in fig.2.

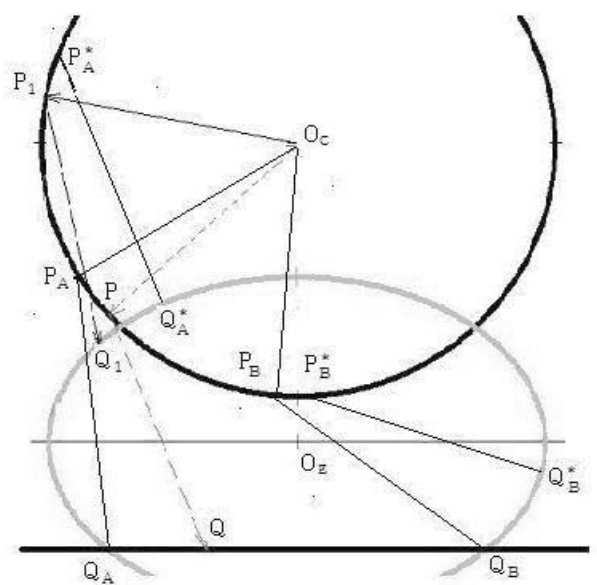

Fig. 2. Physical model of walking robot leg.

Articulated extremity of the leg to robot platform is denoted by $O_{C}$ and the components of the robot leg are segments denoted $O_{C} P$ and $P Q$ of the length $a$ respectively $c$, and where the point $P$ is the knee joint of the leg with the point $Q$ the leg base point.

The knee joint $P$ describe, in cyclic movement of robot leg, a trajectory on circle arc, and a base point $Q$ of the leg an imposed elliptic trajectory on the superior ellipse arc $Q_{B} Q_{A}$.

The circular trajectory is considered with the radius $a$ and centre $O_{C}$ while the elliptic trajectory is assumed with the semiaxes $a, b$ and centre $O_{E}$. The elliptic and respectively circular trajectories are transverse involving impose constant speed component of the leg base point, on horizontal direction. The critical point of the robot leg evolution is the point $P_{c}$ on the circular arc and corresponding $Q_{c}$ point on the elliptic arc with the change of the movement direction of the "knee joint".

The orthogonal system of coordinates for the physical model from fig.2 is identified by the following values of coordinates associated to the figure points:

$$
\begin{array}{r}
O_{C}(a, h), O_{E}\left(a, b_{1}\right), P\left(x_{P}, y_{P}\right), \\
Q\left(x_{Q}, y_{Q}\right), Q_{A}\left(x_{A}, 0\right), Q_{B}\left(x_{B}, 0\right) .
\end{array}
$$

Some conditions are imposed on the model parameters:

$$
a>b>b_{1}>0 ; a+c>h
$$

In critical position of leg "knee joint" evolution and in corresponding leg "base point" position is necessary null speed, important property that will be used in concrete robot leg mathematical modelled evolution. 
The points $P_{A}^{*}$ and $P_{B}^{*}$ define extremities of the maximal domain on the circular arc where the point $P$ can be moved because in this position the segments $P_{A}^{*} Q_{A}^{*}$ and $P_{B}^{*} Q_{B}^{*}$ are normally on the ellipse arc.

The mathematical model of the robot leg deduced from the physical model defined in fig.2 is described by the implicit functions from (1):

$$
\begin{gathered}
(x-a)^{2}+\left(y_{P}-h\right)^{2}-a^{2}=0 . \\
(x-a)^{2} / a^{2}+\left(y_{Q}-b_{1}\right)^{2} / b^{2}=1 .
\end{gathered}
$$

A covering domain for the variables from (1) is defined below:

$$
x \in[0,2 a], y_{P} \in[h-a, h+a), y_{Q} \in\left[0, b+b_{1}\right] .
$$

The explicit functions deduced from (1) have the form:

$$
\begin{gathered}
y_{P}=h \mp\left(2 a x-x^{2}\right)^{1 / 2} ; \\
y_{Q}= \pm b / a\left(2 a x-x^{2}\right)^{1 / 2}+b_{1}
\end{gathered}
$$

Let $P\left(x_{P}, y_{P}\right)$ and $Q\left(x_{Q}, y_{Q}\right)$ be points on the circle arc respectively on the ellipse arc that correspond for one position of the robot leg evolution. The condition $\left(x_{\mathrm{P}}-x_{Q}\right)^{2}+$ $+\left(y_{P}-y_{Q}\right)^{2}-c^{2}=0$ is imposed.

In the triangle $O_{C} P Q$, the segment $O_{C} Q$ can be expressed as function of $\theta$, measure of angle $\left(O_{C} P Q\right)$ :

$$
\left(O_{C} Q\right)^{2}=\left(O_{C} P\right)^{2}+(P Q)^{2}-2\left(O_{C} P\right)(P Q) \cos \theta
$$

The following analytical relation is true, where the variable $x_{Q}$ is considered an independent variable and is denoted by $x$ :

$$
\begin{gathered}
(a-x)^{2}+\left[h-b_{1} \mp(b / a)\left(2 a x-x^{2}\right)^{1 / 2}\right]^{2}= \\
=a^{2}+c^{2}-2 a c \cos \theta
\end{gathered}
$$

The relation (5) can be used to evaluate the measure of the angle $\left(O_{C} P Q\right)$ as function of variable $x$, for $x \in[0,2 a]$. The $Q_{A}\left(x_{A}, 0\right)$ and $Q_{B}\left(x_{B}, 0\right)$ points have abscises $x_{A}=a-$ $-a\left(1-\left(b_{1} / b\right)^{2}\right)^{1 / 2}$ and $x_{B}=a+a\left(1-\left(b_{1} / b\right)^{2}\right)^{1 / 2}$, where $x_{A}$, $x_{B}$ are strictly between 0 and $2 a$.

The uniform linear evolution of the variable $x$ between 0 and $2 a$ is assumed as below, where the selected constant speed $\omega$ and initial condition $x_{0}$ are considered:

$$
x(t)=\omega t+x_{0}
$$

An evolution cycle of the robot leg can start from the point $Q_{B}$, moving on the superior ellipse arc up to point $Q_{A}$, using an evolution law on horizontal axe defined by (6), excepting a neighborhood of critical points.

The covering domain of variables is mentioned below.

$$
x \in[0,2 a], y_{P} \in[h-a, h+a], y_{Q} \in\left[0, b-b_{1}\right]
$$

The explicit functions selected for the physical models from figs. 3 and 4, are as:

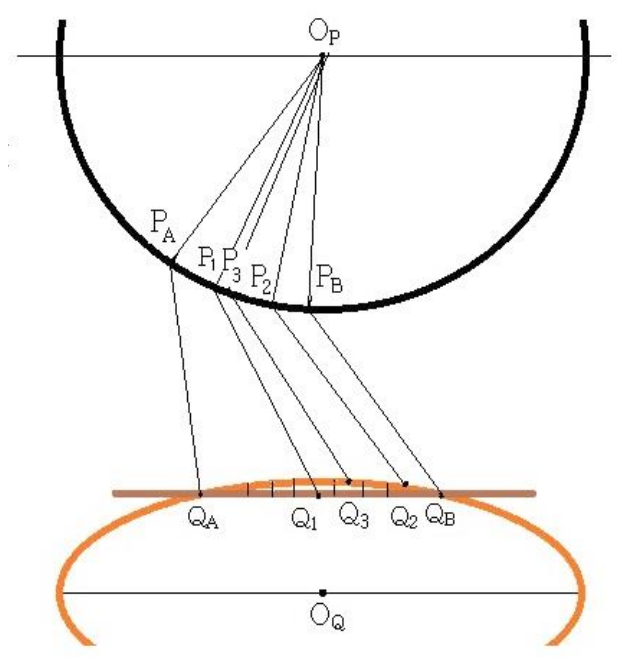

Fg.3. One physical model for walking robot leg.

$$
\begin{gathered}
y_{P}=h-\left(2 a x-x^{2}\right)^{1 / 2} \\
y_{Q}=b / a\left(2 a x-x^{2}\right)^{1 / 2}-b_{1}
\end{gathered}
$$

In first concrete case of walking robot leg physical model is assured the below relation, where $x \in\left[x_{Q A}, x_{Q B}\right]$ :

$$
\begin{gathered}
(a-x)^{2}+\left[(b / a)\left(2 a x-x^{2}\right)^{1 / 2}-\right. \\
\left.-\left(b_{1}+h\right)\right]^{2}=a^{2}+c^{2}-2 a c \cos \theta
\end{gathered}
$$

Because the knee joint speed is natural of zero value in critical positions where the sense of motion is changed, using also Cauchy conditions of continuity is necessary in neighborhood of critical points to assure continue values of displacement and speed as functions of time.

In the following we describe particular cases of walking robot legs using physical models from the figs. 3 and 4 . The walking robot's physical model is defined with the help of the corresponding robot leg definition.

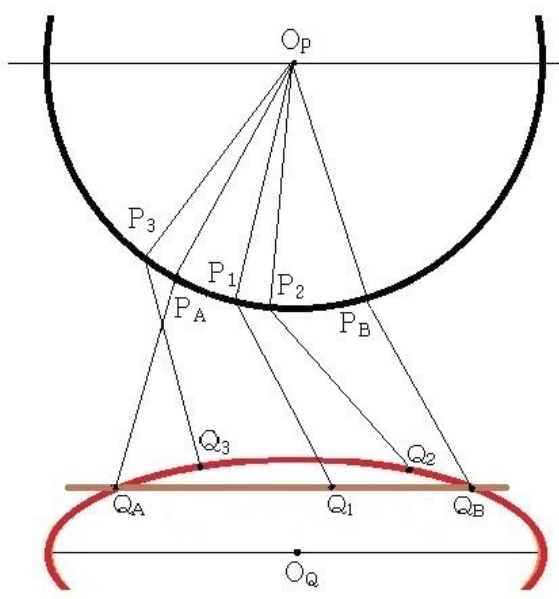

Fig.4. Other physical model of walking robot leg. 
For the physical robot leg model from fig. 3 the pivot point of leg $O_{P}$, the mobile joint $P$ of the leg that describes a circle arc in the leg's evolution on cycle and the point $Q$ of the robot leg base are positioned in our case on an ellipse arc of centre $O_{Q}$ and semi axes length $a$ and $b$. The orthogonal system of coordinate axes is analogue as for fig.2.

Let $O_{P}(a, h), O_{Q}\left(a,-b_{1}\right), Q_{A}\left(x_{A}, 0\right), Q_{B}\left(x_{B}, 0\right)$ be points from figs. 3 and 4.

An evolution cycle of the leg base point from fig.3 is defined by the succession $Q_{A}, Q_{1}, Q_{B}$, on the horizontal axis followed by the succession $Q_{2}, Q_{3}, Q_{A}$ on the superior ellipse arc. The critical points are not on the physical model from fig. 3 but exist on the physical model from fig. 4 .

These two models are different through the value of parameter $h$, in the first case the value $h_{1}$ and in the second case the value $h_{2}>h_{1}$ that imply the corresponding modification of the parameter $b_{1}$.

The conditions $a>b>b_{1}>0 ; a+c>h$ are assumed, where the length of the inferior component of the robot leg is $c$. For the physical model from fig. 3 is assumed $c>a$.

Our critical position definition in the robot leg evolution, reminded here by us, is the position where the point $P_{c}$ on the circular arc and corresponding $Q_{c}$ point on the elliptic arc have the property of changing the movement direction of the "knee joint".

In the critical position of leg "knee joint" evolution and in corresponding leg "base point" evolution we remark the necessary null speed of leg "knee joint" and we mention again that this is an important property used in assurance of concrete evolution of the robot leg.

The critical points $P_{c}$ and $Q_{c}$, in our concrete case, define extremities of one maximal domain on the circular arc where the point $P$ can be moved and in this position the segment $P_{c} Q_{c}$ is normally on the ellipse arc. The sense of movement is changed on the circle arc in the extremities of the continuous domain of evolution for "knee joint". In our opinion this notion on critical position can be generalized in the case more general of walking robot movement where the ellipse arc can be substituted by another continuous curve.

The analytical method for identification of critical points on the circle arc and implicit the critical points on the ellipse arc, for concrete physical model from fig.4, is deduced by solving the equation that depend on $x_{Q}$ imposed by the orthogonally condition.

The ellipse arc trajectory of walking robot leg base point is recommended by us for walking up stairs in kinematics or possible dynamics of walking robot evolution (see fig.5). The stair is configured in vertical plane, by broken line $A B C D$.

The walking robot can be imagined as in fig.1. The trajectory of one leg, in cyclic climbing by stairs of the walking robot is assured ellipse arc $E F$, where the points $E, F$ are selected critical points of the leg movement.

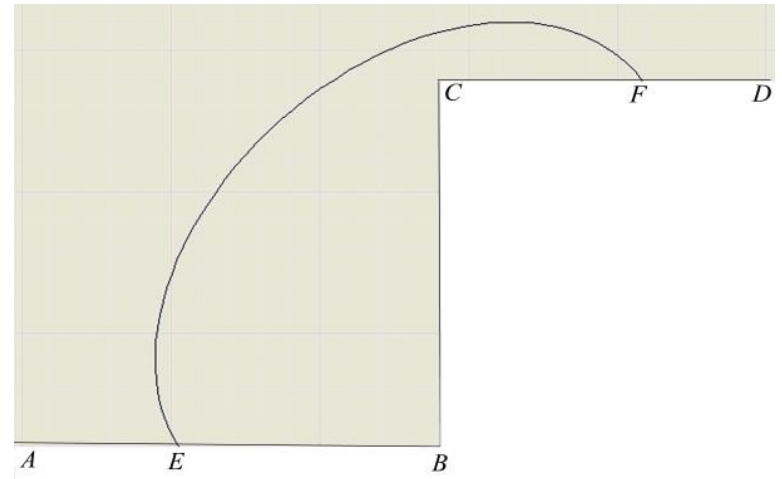

Fig. 5. Recommended trajectories of leg base point for walking up stairs

In the critical points $E, F$, is necessary to be assured zero value of leg base point speed. The synchronized evolution of the legs is searched so that the stability (existence) of the walking robot to be assured.

We remark that the property of walking robot stability (existence) position is also maintained in a neighborhood for one such position.

\section{CONCLUSIONS}

A mathematical study on the walking robots dynamic system stability control possibilities, the walking robot being considered as particular case of a dynamic (kinematics) system that depends on parameters, is performed. The mathematical conditions on the separation between stable and unstable regions, in the domain of the dynamic system free parameters, discovered by us, are emphasized, calling the algebraic operations in complex domain that permit new results in the stability theory.

The property of separation described in the paper, encountered also in many other dynamic systems from the literature, without mathematical justification, is very important because it creates the possibility of the stability control of a dynamic system by optimization of the system evolution using the compatible criterion in the stability regions of the free parameters domain. The study for the dynamic systems can be performed, with conservation of the fundamental idea, and for kinematics of the walking robots. A defined walking robot having the property of separation in stable and unstable regions is analyzed. The property of stable region separation is described in our case of walking robot leg with the possibility to be extended on other cases. The critical position on the particular case of the walking robot is defined in the paper and a mathematical method of its identification is performed. The possibilities of our study application in control of walking robot evolution can be as example for walking up stairs that is underlined in the paper. We remark that our study has not exhausted the problem of dynamical systems stability control or for kinematics stability control and that however an interesting domain of walking robot stable evolution control has been opened. 


\section{ACKNOWLEDGMENT}

This work was developed with the support of MENUEFISCDI, PN-II-PT-PCCA-2013-4, VIPRO project No.009/2014, Romanian Academy and "Joint Laboratory of Intelligent Rehabilitation Robot" collaborative research agreement between Romanian Academy by IMSAR and Yanshan University, China, project KY201501009, 2015.

\section{REFERENCES}

[1] Hirsch, M. W., Smale, S.,and Devaney, R. L. Differential Equations, Dynamical Systems, and an Introduction to Chaos, Academic Press, Amsterdam, Boston, Heidelberg, London, New York, Oxford, Paris, San Diego, San Francisco, Singapore, Sydney, Tokyo (2004).

[2] Halanay, A., and Rasvan, V. Applications of Liapunov Methods in Stability, Kluwer Academic Publishers, Dordrecht, Boston, London, (1993).

[3] Hayashi, C. Nonlinear Oscillations in Physical Systems, Ed. McGraw Hill Book Company, New York, San Francisco, Toronto, London, (1964).

[4] Voinea R.P. and Stroe I.V. Introduction in the Theory of Dynamical Systems (in Romanian) Academy Printing House, Bucharest (2000).

[5] Zeveleanu C. and Bratu P. Nonlinear Vibrations (in Romanian), Impuls Printing House, Bucharest (2001).

[6] Teodorescu P.P., Stanescu N.D., Pandrea N., Numerical analysis with applications in mechanics and engineering, John Wiley \& Sons, Hoboken, USA, (2013).

[7] Stanescu N.D., Munteanu L., Chiroiu V. and Pandrea N. Dynamical Systems. Theory and Applications (in Romanian), Romanian Academy Printing House, Bucharest, Vol.I (2007), Vol.II (2011).

[8] Lazăr, D. Analytical Mechanics Principles (in Romanian), Technical Printing House, Bucharest, (1976).

[9] Hacker, T. Stability and command in flight theory (in Romanian), Academy Printing House, Bucharest, (1968).

[10] Jung-Yup Kim, Ill-Woo Park, Jun-Ho Oh, Walking Control Algorithm of Biped Humanoid Robot on Uneven and Inclined Floor, Springer Science, J. Intell Robot Syst ; 48:457-484, DOI 10.1007/s10846-0069107-829-65, (2007).

[11] Vladareanu, L., Tont, G., Ion, I., Munteanu, M. S., Mitroi, D., Walking Robots Dynamic Control Systems on an Uneven Terrain, Advances in Electrical and Computer Engineering, 10(2), 146-153, (2010).

[12] Migdalovici, M., Baran, D., Vladeanu G., Dynamical systems stability. Theory and applications (in Romanian), Self-Publishing Printing House, Bucharest, (2013).

[13] Migdalovici, M., Baran, D., On a method of vibration analysis for dynamical systems, Topics in Applied Mechanics, Eds. Chiroiu V. and
Sireteanu T., Romanian Academy Printing House, Bucharest, I, Cap.9, 248-266, (2003),

[14] Migdalovici, M. and Baran, D. About the stability of motion for two sprung superposed masses in contact with a wire, Proceedings ICSV10, Stockholm, 2147-2154, (2003).

[15] Migdalovici, M. and Baran, D. On a stability analysis method of dynamical systems with numerical tests, Proceedings of the Int. Conf. of diff. geom. and dynamical systems (DGDS-2008), Mangalia, Romania, Geometry Balkan Press, 99-107, (2009).

[16] Chung, Y. I. and Genin, J. Stability of a vehicle on a multispan simply supported guideway, J. of Dynamic Systems, Measurements and Control, 100, 326-331, (1978).

[17] Poetsch, G. and all. Pantograph / catenary dynamics and control, Vehicle system dynamics, 28, 159-195, (1997).

[18] Migdalovici, M., Baran, D., Vladeanu, G. On the separation conditions of the stability zones of the dynamical systems that depend of parameters, ICSV20, Bangkok, Proceedings on CD, (2013).

[19] Migdalovici, M., Baran, D., Vladeanu, G. Mathematical aspects on the stability of nonlinear dynamical systems, ICSV21, Beijing, Proceedings on $\mathrm{CD},(2014)$.

[20] Lupu M. and Isaia F. The study of some nonlinear dynamical systems modelled by a more general Rayleigh-Van Der Pol equation, Creative Math. \& Inf., 16, 81-90, (2007).

[21] Kumaniecka, A. and Grzyb, A., On dynamics of catenary - pantograph modelled as discrete continuous system, Machine dynamics problems, 24, 105-119, (2000).

[22] Staretu I., Itu A.M. and Moldovan C.C. Software Modules for Command and Control of the Anthropomorphic Gripper for Robots, Applied Mechanics and Materials, 332, 229-234, (2013).

[23] Migdalovici M., Sireteanu T, and Videa E.M. Control of Vibration of Transmission Lines, International Journal of Acoustics and Vibration, 15(2), 65-71, (2010).

[24] Wilkinson, J. H. Convergence of the LR, QR and related algorithms, Computer J. 8, 77-84, (1965).

[25] Rutishauser, H., Solution of eigenvalue problems with the LR transformation, Appl. Math Ser., Nat. Bur. Stand., 49, 47-81, (1958).

[26] Rutishauser, H. and Bauer, F. L. Determination des vecteurs propres d'une matrice par une methode iterative avec convergence quadratique, C.R. Acad. Sci., Paris, 240, 1680, (1955)

[27] Francis, J. G. F., QR transformation, The Computer Journal, 4, First part, 265-271, (1961), Second part, 332-345, (1962).

[28] Parlet, B.N. Global convergence of the basic QR algorithm on Hessenberg matrices, Math. of Computation, 22, 803-817, (1968)

[29] Martin R.S., Peters G. and Wilkinson J.H. The QR Agorithm for Real Hessenberg Matrices, Numer. Math. 14, 219-231, (1970) 